\title{
A NADLER-TYPE FIXED POINT THEOREM IN DISLOCATED SPACES AND APPLICATIONS
}

\author{
H. AYDI, A. FELHI, ERDAL KARAPINAR, AND S. SAHMIM
}

Received 16 October, 2015

\begin{abstract}
In this paper, we introduce the concept of a Hausdorff dislocated metric. We initiate the study of fixed point theory for multi-valued mappings on dislocated metric space using the Hausdorff dislocated metric and we prove a generalization of the well known Nadler's fixed point theorem. Moreover, we provide some examples and we give an application of our main result.
\end{abstract}

2010 Mathematics Subject Classification: 47H10; 54H25

Keywords: Hausdorff dislocated metric, multi-valued mapping, Nadler's fixed point theorem

\section{INTRODUCTION AND PRELIMINARIES}

Let $(X, d)$ be a metric space and $C B(X)$ denotes the collection of all nonempty closed and bounded subsets of $X$. For $A, B \in C B(X)$, define

$$
H(A, B):=\max \left\{\sup _{a \in A} d(a, B), \sup _{b \in B} d(b, A)\right\},
$$

where $d(x, A):=\inf \{d(x, a): a \in A\}$ is the distance of a point $x$ to the set $A$. It is known that $H$ is a metric on $C B(X)$, called the Hausdorff metric induced by the metric $d$.

Definition 1. Let $X$ be any nonempty set. An element $x$ in $X$ is said to be a a fixed point of a multi-valued mapping $T: X \rightarrow 2^{X}$ if $x \in T x$, where $2^{X}$ denotes the collection of all nonempty subsets of $X$. if

We recall that a multi-valued mapping $T: X \rightarrow C B(X)$ is said to be a contraction

$$
H(T x, T y) \leq k d(x, y)
$$

for all $x, y \in X$ and for some $k$ in $[0,1)$.

The study of fixed points for multi-valued contractions using the Hausdorff metric was initiated by Nadler [18] who proved the following theorem.

Theorem 1 ([18]). Let $(X, d)$ be a complete metric space and $T: X \rightarrow C B(X)$ be a contraction mapping. Then, there exists $x \in X$ such that $x \in T x$. 
The notion of dislocated metric space was introduced by Hitzler and Seda [12] (see also [11] ). Later, Amini-Harandi [9] re-discovered the notion of dislocated metric under the name of "metric-like". In this paper, the author [9] presented some fixed point results in the class of dislocated metric spaces. Very recently, Karapinar and Salimi [19] established some fixed point theorems for cyclic contractions. For more fixed point results on dislocated metric spaces, see e.g. [1-3, 7, 8, 13, 15, 16, 20-23].

Definition 2. Let $X$ be a nonempty set. A function $\sigma: X \times X \rightarrow[0, \infty)$ is said to be a dislocated metric (or a metric-like) on $X$ if for any $x, y, z \in X$, the following conditions hold:

$\left(\sigma_{1}\right) \sigma(x, y)=0 \Longrightarrow x=y$;

$\left(\sigma_{2}\right) \sigma(x, y)=\sigma(y, x)$

$\left(\sigma_{3}\right) \sigma(x, z) \leq \sigma(x, y)+\sigma(y, z)$.

The pair $(X, \sigma)$ is then called a dislocated metric (metric-like) space.

It is known that a partial metric [17] is also a dislocated metric. So, a trivial example of a dislocated metric space is the pair $([0, \infty), \sigma)$, where $\sigma:[0, \infty) \times[0, \infty) \rightarrow[0, \infty)$ is defined as $\sigma(x, y)=\max \{x, y\}$.

In the sequel, $\mathbb{R}_{0}^{+}$represents the set of all nonnegative reals. In the following example, we give a dislocated metric which is neither a metric nor a partial metric.

Example 1 ([6]). Take $X=\{1,2,3\}$ and consider the dislocated metric $\sigma: X^{2} \rightarrow$ $\mathbb{R}_{0}^{+}$given by

$$
\begin{gathered}
\sigma(1,1)=0, \quad \sigma(2,2)=1, \quad \sigma(3,3)=\frac{2}{3}, \\
\sigma(1,2)=\sigma(2,1)=\frac{9}{10}, \quad \sigma(2,3)=\sigma(3,2)=\frac{4}{5}, \\
\sigma(1,3)=\sigma(3,1)=\frac{7}{10} .
\end{gathered}
$$

Since $\sigma(2,2) \neq 0, \sigma$ is not a metric and since $\sigma(2,2)>\sigma(1,2), \sigma$ is not a partial metric [17].

Each dislocated metric $\sigma$ on $X$ generates a $T_{0}$ topology $\tau_{\sigma}$ on $X$ which has as a base the family open $\sigma$-balls $\left\{B_{\sigma}(x, \varepsilon): x \in X, \varepsilon>0\right\}$, where $B_{\sigma}(x, \varepsilon)=\{y \in X$ : $|\sigma(x, y)-\sigma(x, x)|<\varepsilon\}$, for all $x \in X$ and $\varepsilon>0$.

Observe that a sequence $\left\{x_{n}\right\}$ in a dislocated metric space $(X, \sigma)$ converges to a point $x \in X$, with respect to $\tau_{\sigma}$, if and only if $\sigma(x, x)=\lim _{n \rightarrow \infty} \sigma\left(x, x_{n}\right)$.

Definition 3. Let $(X, \sigma)$ be a dislocated metric space.

(a) A sequence $\left\{x_{n}\right\}$ in $X$ is said to be a Cauchy sequence if $\lim _{n, m \rightarrow \infty} \sigma\left(x_{n}, x_{m}\right)$ exists and is finite. 
(b) $(X, \sigma)$ is said to be complete if every Cauchy sequence $\left\{x_{n}\right\}$ in $X$ converges with respect to $\tau_{\sigma}$ to a point $x \in X$ such that $\lim _{n \rightarrow \infty} \sigma\left(x, x_{n}\right)=\sigma(x, x)=$ $\lim _{n, m \rightarrow \infty} \sigma\left(x_{n}, x_{m}\right)$.

We need in the sequel the following trivial inequality

$$
\sigma(x, x) \leq 2 \sigma(x, y) \text { for all } x, y \in X .
$$

In this paper, we introduce a new concept called a Hausdorff dislocated metric . Using this concept, we establish a fixed point result for multi-valued mappings involving a generalized contraction. We derive many interesting corollaries on existing known results in the literature. Our obtained results are supported by some examples and an application to an integral equation.

\section{HAUSDORFF DISLOCATED METRIC}

Let $(X, \sigma)$ be a dislocated metric space. Let $C B^{\sigma}(X)$ be the family of all nonempty, closed and bounded subsets in the dislocated metric space $(X, \sigma)$, induced by the dislocated metric $\sigma$. Note that the boundedness is given as follows: $A$ is a bounded subset in $(X, \sigma)$ if there exist $x_{0} \in X$ and $M \geq 0$ such that for all $a \in A$, we have $a \in B_{\sigma}\left(x_{0}, M\right)$, that is,

$$
\left|\sigma\left(x_{0}, a\right)-\sigma\left(x_{0}, x_{0}\right)\right|<M .
$$

The Closedness is taken in $\left(X, \tau_{\sigma}\right)$ (where $\tau_{\sigma}$ is the topology induced by $\sigma$ ). Let $\bar{A}$ be the closure of $A$ with respect to the dislocated metric $\sigma$. We have

\section{Definition 4.}

$$
\begin{aligned}
a \in \bar{A} & \Longleftrightarrow B_{\sigma}(a, \varepsilon) \cap A \neq \varnothing \quad \text { for all } \varepsilon>0 \\
& \Longleftrightarrow \text { there exists } x_{n} \in A, \quad x_{n} \rightarrow a \text { in }(X, \sigma) .
\end{aligned}
$$

If $A \in C B^{\sigma}(X)$, then $\bar{A}=A$.

For $A, B \in C B^{\sigma}(X)$ and $x \in X$, define

$$
\begin{aligned}
\sigma(x, A) & =\inf \{\sigma(x, a), a \in A\}, \delta_{\sigma}(A, B)=\sup \{\sigma(a, B): a \in A\} \quad \text { and } \\
\delta_{\sigma}(B, A) & =\sup \{\sigma(b, A): b \in B\} .
\end{aligned}
$$

Lemma 1. Let $(X, \sigma)$ be a dislocated metric space and $A$ be any nonempty set in $(X, \sigma)$, then

$$
\text { if } \sigma(a, A)=0, \quad \text { then } a \in \bar{A} .
$$

Also, if $\left\{x_{n}\right\}$ is a sequence in $(X, \sigma)$ that is $\tau_{\sigma}$-convergent to $x \in X$, then

$$
\lim _{n \rightarrow \infty}\left|\sigma\left(x_{n}, A\right)-\sigma(x, A)\right|=\sigma(x, x) .
$$


Proof. If $\sigma(a, A)=0$, so $\inf _{x \in A} \sigma(a, x)=0$, that is, for all $\varepsilon>0$, there exists $x \in A$ such that $\sigma(a, x)<\varepsilon$. Hence, for all $n \geq 1$, there exists $x_{n} \in A$ such that

$$
\sigma\left(a, x_{n}\right)<\frac{1}{n}
$$

Thus, $\lim _{n \rightarrow \infty} \sigma\left(a, x_{n}\right)=0$. By (1.1), we have $\sigma(a, a) \leq 2 \sigma\left(a, x_{n}\right), \forall n$.

Then, $\sigma(a, a) \leq 2 \lim _{n \rightarrow \infty} \sigma\left(a, x_{n}\right)=0$. Finally, we obtain $\lim _{n \rightarrow \infty} \sigma\left(a, x_{n}\right)=\sigma(a, a)=0$, which means that $\left\{x_{n}\right\}$ converges to $a$ in $(X, \sigma)$. By Definition $4, a \in \bar{A}$.

The equality from (2.2) follows from the inequality

$$
\left|\sigma\left(x_{n}-A\right)-\sigma(x, A)\right|=\sigma\left(x_{n}, x\right) .
$$

Remark 1. It was shown in Remark 2.1 from [4] that if $A$ is a subset of a partial metric space $(X, p)$ and $x \in X$, then

$$
x \in \bar{A} \Longleftrightarrow p(x, A)=p(x, x) .
$$

We show by an example that this property is not longer true in a dislocated metric space.

Example 2. Let $X=\{0,1\}$ and $\sigma: X \times X \rightarrow \mathbb{R}_{0}^{+}$be defined by

$$
\sigma(0,0)=2 \text { and } \sigma(x, y)=1 \text { if }(x, y) \neq(0,0) .
$$

Then, $(X, \sigma)$ is a dislocated metric space. Note that $\sigma$ is not a partial metric on $X$ because $\sigma(0,0) \geq \sigma(1,0)$.

We have $0 \in \bar{X}(=X)$, but $\sigma(0, X)=\min \{\sigma(0,0), \sigma(0,1))=1 \neq \sigma(0,0)$

Let $(X, \sigma)$ be a dislocated metric space. For $A, B \in C B^{\sigma}(X)$, define

$$
H_{\sigma}(A, B)=\max \left\{\delta_{\sigma}(A, B), \delta_{\sigma}(B, A)\right\} .
$$

Now, we shall study some properties of $H_{\sigma}: C B^{\sigma}(X) \times C B^{\sigma}(X) \rightarrow[0, \infty)$.

Proposition 1. Let $(X, \sigma)$ be a dislocated metric space. For all $A, B, C \in C B^{\sigma}(X)$, we have the following:

$$
\begin{aligned}
(\text { i }): H_{\sigma}(A, A) & =\delta_{\sigma}(A, A)=\sup \{\sigma(a, A): a \in A\} ; \\
\text { (ii }): H_{\sigma}(A, B) & =H_{\sigma}(B, A) ; \\
\text { (ii i }): H_{\sigma}(A, B) & =0 \text { implies that } A=B ; \\
\text { (iv) }: H_{\sigma}(A, B) & \leq H_{\sigma}(A, C)+H_{\sigma}(C, B) .
\end{aligned}
$$

Proof. (i) and (ii) are clear.

(iii) Suppose that $H_{\sigma}(A, B)=0$. Then,

$$
\sup _{a \in A} \sigma(a, B)=0 .
$$


Mention that $\sup \sigma(a, B)=0$, implies $\forall a \in A, \sigma(a, B)=0$. Then, by lemma 1 , $a \in \bar{B}=B$. As $a$ is arbitrary in $A$, we conclude that $A \subset B$.

Similarly, $H_{\sigma}(B, A)=0$ implies $B \subset A$.

(iv) Let $a \in A, b \in B$ and $c \in C$. As

$$
\sigma(a, b) \leq \sigma(a, c)+\sigma(c, b)
$$

so we have

$$
\sigma(a, B) \leq \sigma(a, c)+\sigma(c, B) \leq \sigma(a, c)+\delta_{\sigma}(C, B) \leq \sigma(a, C)+\delta_{\sigma}(C, B),
$$

since $c$ is an arbitrary element of $C$. As $a$ is an arbitrary element of $A$, it follows

$$
\delta_{\sigma}(A, B) \leq \delta_{\sigma}(A, C)+\delta_{\sigma}(C, B) \leq H_{\sigma}(A, C)+H_{\sigma}(C, B) .
$$

Similarly, due to symmetry of $H_{\sigma}$, we have

$$
\delta_{\sigma}(B, A) \leq H_{\sigma}(A, C)+H_{\sigma}(C, B) .
$$

Combining the two above inequalities, we get (iv).

Remark 2. The converse of assertion ( $i$ i i ) from Proposition 1 is not true in general as it is clear from the following example.

Example 3. Let $X=\{0,1\}$ be endowed with the dislocated metric $\sigma: X \times X \rightarrow$ $[0, \infty)$ defined by

$$
\sigma(1,1)=2 \quad \text { and } \sigma(0,0)=\sigma(0,1)=\sigma(1,0)=1 .
$$

Note that $\sigma$ is not a partial metric since $\sigma(1,1)>\sigma(1,0)$. From (i) of Proposition 1, we have

$$
\begin{aligned}
H_{\sigma}(X, X)=\delta_{\sigma}(X, X) & =\sup \{\sigma(x, X), x \in\{0,1\}\} \\
& =\max \{\sigma(0,\{0,1\}), \sigma(1,\{0,1\})\}=1 \neq 0 .
\end{aligned}
$$

In view of Proposition 1, we call the mapping $H_{\sigma}: C B^{\sigma}(X) \times C B^{\sigma}(X) \rightarrow[0,+\infty)$, a Hausdorff dislocated metric induced by $\sigma$.

Remark 3. It is easy to show that any Hausdorff metric is a Hausdorff dislocated metric. The converse is not true (see Example 3).

\section{FIXED POINT OF MULTI-VALUED CONTRACTION MAPPINGS}

We start with the following simple useful lemma. One may find its analogous for the partial metric case in [5].

Lemma 2. Let $A, B \in C B^{\sigma}(X)$ and $a \in A$. Then, for all $\varepsilon>0$, there exists a point $b \in B$ such that $\sigma(a, b) \leq H_{\sigma}(A, B)+\varepsilon$. 
The inequality from Lemma 2 also appears in Nadler's paper [18]. Now, we state and prove our main result.

Theorem 2. Let $(X, \sigma)$ be a complete dislocated metric space. If $T: X \rightarrow C B^{\sigma}(X)$ is a multi-valued mapping such that for all $x, y \in X$, we have

$$
H_{\sigma}(T x, T y) \leq k M(x, y)
$$

where $k \in[0,1)$ and

$$
M(x, y)=\max \left\{\sigma(x, y), \sigma(x, T x), \sigma(y, T y), \frac{1}{4}(\sigma(x, T y)+\sigma(y, T x))\right\} .
$$

Then, $T$ has a fixed point.

Proof. Let $x_{0} \in X$ and $x_{1} \in T x_{0}$. Clearly, if $\sigma\left(x_{0}, x_{1}\right)=0$, then $x_{0}=x_{1}$ and $x_{0}$ is a fixed point of $T$. Assume $\sigma\left(x_{0}, x_{1}\right)>0$. Since $T x_{0}, T x_{1} \in C B^{\sigma}(X)$ and $x_{1} \in T x_{0}$, Lemma 2 implies the existence of a point $x_{2} \in T x_{1}$ such that

$$
\sigma\left(x_{2}, x_{1}\right) \leq H_{\sigma}\left(T x_{1}, T x_{0}\right)+\frac{1-k}{2} M\left(x_{1}, x_{0}\right) .
$$

If $\sigma\left(x_{2}, x_{1}\right)=0$, then $x_{2}=x_{1}$ and $x_{1}$ is a fixed point of $T$. Assuming $\sigma\left(x_{2}, x_{1}\right)>0$, then, by Lemma 2, there is a point $x_{3} \in T x_{2}$ such that

$$
\sigma\left(x_{3}, x_{2}\right) \leq H_{\sigma}\left(T x_{2}, T x_{1}\right)+\frac{1-k}{2} M\left(x_{2}, x_{1}\right) .
$$

Continuing in this fashion, we complete a sequence $\left(x_{n}\right) \subset X$ such that $x_{n+1} \in T x_{n}$ and $\sigma\left(x_{n}, x_{n+1}\right)>0$ with

$$
\sigma\left(x_{n+1}, x_{n}\right) \leq H_{\sigma}\left(T x_{n}, T x_{n-1}\right)+\frac{1-k}{2} M\left(x_{n}, x_{n-1}\right) .
$$

Then, we get

$$
\begin{aligned}
& \sigma\left(x_{n+1}, x_{n}\right) \\
\leq & k M\left(x_{n}, x_{n-1}\right)+\frac{1-k}{2} M\left(x_{n}, x_{n-1}\right) \\
= & \frac{1+k}{2} M\left(x_{n}, x_{n-1}\right) \\
\leq & \frac{1+k}{2} \max \left\{\sigma\left(x_{n}, x_{n-1}\right), \sigma\left(x_{n}, x_{n+1}\right), \frac{1}{4}\left[\sigma\left(x_{n}, x_{n}\right)+\sigma\left(x_{n-1}, x_{n+1}\right)\right]\right\} .
\end{aligned}
$$

By a triangular inequality, we get

$$
\begin{aligned}
\frac{1}{4}\left(\sigma\left(x_{n}, x_{n}\right)+\sigma\left(x_{n-1}, x_{n+1}\right)\right) & \leq \frac{1}{4}\left(3 \sigma\left(x_{n}, x_{n-1}\right)+\sigma\left(x_{n+1}, x_{n}\right)\right) \\
& \leq \max \left\{\sigma\left(x_{n}, x_{n-1}\right), \sigma\left(x_{n}, x_{n+1}\right)\right\} .
\end{aligned}
$$


Then

$$
\sigma\left(x_{n}, x_{n+1}\right) \leq \frac{1+k}{2} \max \left\{\sigma\left(x_{n-1}, x_{n}\right), \sigma\left(x_{n}, x_{n+1}\right)\right\} .
$$

Now, if $\sigma\left(x_{n}, x_{n+1}\right)>\sigma\left(x_{n-1}, x_{n}\right)$, then we have

$$
\sigma\left(x_{n}, x_{n+1}\right) \leq \frac{1+k}{2} \sigma\left(x_{n}, x_{n+1}\right)<\sigma\left(x_{n}, x_{n+1}\right),
$$

which is a contradiction. So, for all $n \geq 1, \sigma\left(x_{n}, x_{n+1}\right) \leq \sigma\left(x_{n}, x_{n-1}\right)$. Finally, we get

$$
\sigma\left(x_{n}, x_{n+1}\right) \leq \frac{1+k}{2} \sigma\left(x_{n-1}, x_{n}\right), \forall n \geq 1 .
$$

Moreover, by induction, one finds

$$
\sigma\left(x_{n}, x_{n+1}\right) \leq\left(\frac{1+k}{2}\right)^{n} \sigma\left(x_{0}, x_{1}\right), \forall n \geq 1 .
$$

Since $k \in[0,1)$, we have $\sum_{n \geq 0}\left(\frac{1+k}{2}\right)^{n}<\infty$. So, for all $p \geq 0$, we have

$$
\begin{aligned}
\sigma\left(x_{n}, x_{n+p}\right) & \leq \sigma\left(x_{n}, x_{n+1}\right)+\sigma\left(x_{n+1}, x_{n+2}\right)+\ldots+\sigma\left(x_{n+p-1}, x_{n+p}\right) \\
& \leq \sum_{i=n}^{n+p-1}\left(\frac{1+k}{2}\right)^{i} \sigma\left(x_{0}, x_{1}\right) \\
& \leq \sum_{i=n}^{\infty}\left(\frac{1+k}{2}\right)^{i} \sigma\left(x_{0}, x_{1}\right) \rightarrow 0 \text { as } n \rightarrow \infty
\end{aligned}
$$

Thus, by symmetry of $\sigma$, we obtain

$$
\lim _{n, m \rightarrow \infty} \sigma\left(x_{n}, x_{m}\right)=0 .
$$

This yields that the sequence $\left\{x_{n}\right\}$ is Cauchy. Since $(X, \sigma)$ is complete, the sequence $\left\{x_{n}\right\}$ converges to a point $x^{\star} \in X$, i.e,

$$
\lim _{n \rightarrow \infty} \sigma\left(x_{n}, x^{\star}\right)=\sigma\left(x^{\star}, x^{\star}\right)=\lim _{n, m \rightarrow \infty} \sigma\left(x_{n}, x_{m}\right)=0 .
$$

We have $\sigma\left(x^{\star}, T x^{\star}\right) \leq \sigma\left(x^{\star}, x_{n+1}\right)+\sigma\left(x_{n+1}, T x^{\star}\right)$.

Since $x_{n+1} \in T x_{n}$, it follows

$$
\begin{aligned}
\sigma\left(x^{\star}, T x^{\star}\right) & \leq \sigma\left(x^{\star}, x_{n+1}\right)+\delta_{\sigma}\left(T x_{n}, T x^{\star}\right) \\
& \leq \sigma\left(x^{\star}, x_{n+1}\right)+H_{\sigma}\left(T x_{n}, T x^{\star}\right) \\
& \leq \sigma\left(x^{\star}, x_{n+1}\right)+k M\left(x_{n}, x^{\star}\right),
\end{aligned}
$$

where

$$
M\left(x_{n}, x^{\star}\right)
$$




$$
=\max \left\{\sigma\left(x_{n}, x^{\star}\right), \sigma\left(x_{n}, T x_{n}\right), \sigma\left(x^{\star}, T x^{\star}\right), \frac{1}{4}\left(\sigma\left(x_{n}, T x^{\star}\right)+\sigma\left(x^{\star}, T x_{n}\right)\right)\right\} .
$$

We have

$$
\begin{gathered}
\sigma\left(x_{n}, T x_{n}\right) \leq \sigma\left(x_{n}, x_{n+1}\right), \\
\sigma\left(x^{\star}, T x_{n}\right) \leq \sigma\left(x^{\star}, x_{n+1}\right) .
\end{gathered}
$$

When passing to limit, it should be mentioned that, by Lemma 1 and (3.6),

$$
\sigma\left(x^{\star}, T x_{n}\right) \rightarrow \sigma\left(x^{\star}, T x^{\star}\right) .
$$

Again, by taking $n \rightarrow \infty$ and using (3.6), we obtain

$$
\begin{aligned}
\sigma\left(x^{\star}, T x^{\star}\right) & \leq k \max \left\{\sigma\left(x^{\star}, T x^{\star}\right), \frac{1}{4} \sigma\left(x^{\star}, T x^{\star}\right)\right\} \\
& =k \sigma\left(x^{\star}, T x^{\star}\right) .
\end{aligned}
$$

Since, $k \in[0,1)$, we have $\sigma\left(x^{\star}, T x^{\star}\right)=0$. Finally, by lemma 1, we have $x^{\star} \in \overline{T x^{\star}}=$ $T x^{\star}$. Then, $x^{\star}$ is a fixed point of $T$.

As consequences of our main result, we may state the following immediate corollaries.

Corollary 1 (Hardy-Rogers type [10]). Let $(X, \sigma)$ be a complete dislocated metric space. If $T: X \rightarrow C B^{\sigma}(X)$ is a multi-valued mapping such that for all $x, y \in X$, we have

$$
H_{\sigma}(T x, T y) \leq a \sigma(x, y)+b \sigma(x, T x)+c \sigma(y, T y)+d[\sigma(x, T y)+\sigma(y, T x)]
$$

where $a, b, c, d \in[0,1)$ such that $a+b+c+4 d<1$. Then, $T$ has a fixed point.

Corollary 2 (Kannan type [14]). Let $(X, \sigma)$ be a complete dislocated metric space. If $T: X \rightarrow C B^{\sigma}(X)$ is a multi-valued mapping such that for all $x, y \in X$, we have

$$
H_{\sigma}(T x, T y) \leq a \sigma(x, y)+b \sigma(x, T x)+c \sigma(y, T y)
$$

where $a, b, c \in[0,1)$ such that $a+b+c<1$. Then, $T$ has a fixed point.

Corollary 3. Let $(X, \sigma)$ be a complete dislocated metric space. If $T: X \rightarrow$ $C B^{\sigma}(X)$ is a multi-valued mapping such that for all $x, y \in X$, we have

$$
H_{\sigma}(T x, T y) \leq k \sigma(x, y)
$$

where $k \in[0,1)$. Then, $T$ has a fixed point.

Corollary 4 ([4]). Let $(X, \sigma)$ be a complete partial metric space. If $T: X \rightarrow$ $C B^{\sigma}(X)$ is a multi-valued mapping such that for all $x, y \in X$, we have

$$
H_{\sigma}(T x, T y) \leq k \sigma(x, y)
$$

where $k \in[0,1)$. Then, $T$ has a fixed point. 
Corollary 5 ([18]). Let $(X, \sigma)$ be a complete metric space. If $T: X \rightarrow C B^{\sigma}(X)$ is a multi-valued mapping such that for all $x, y \in X$, we have

$$
H_{\sigma}(T x, T y) \leq k \sigma(x, y)
$$

where $k \in[0,1)$. Then, $T$ has a fixed point.

Corollary 6. Let $(X, \sigma)$ be a complete dislocated metric space. If $T: X \rightarrow X$ is a single-valued mapping such that for all $x, y \in X$, we have

$$
\begin{gathered}
\sigma(T x, T y) \\
\leq k \max \left\{\sigma(x, y), \sigma(x, T x), \sigma(y, T y), \frac{1}{4}(\sigma(x, T y)+\sigma(y, T x))\right\}
\end{gathered}
$$

where $k \in[0,1)$. Then, $T$ has a fixed point $x \in X$, that is, $T x=x$.

\section{EXAMPLES AND AN APPLICATION}

First, we give the following illustrative examples where the main result of Aydi et al. [4] is not applicable.

Example 4. Let $X=\{0,1,2\}$ and $\sigma: X \times X \rightarrow[0, \infty)$ defined by

$$
\begin{array}{ll}
\sigma(0,0)=\sigma(1,1)=0, & \sigma(2,2)=\frac{23}{48} \\
\sigma(0,1)=\sigma(1,0)=\frac{1}{3}, \quad \sigma(0,2)=\sigma(2,0)=\frac{11}{24} \quad \text { and } \quad \sigma(1,2)=\sigma(2,1)=\frac{1}{2} .
\end{array}
$$

Then, $(X, \sigma)$ is a complete dislocated metric space. Note that $\sigma$ is not a partial metric on $X$ because $\sigma(2,2) \geq \sigma(2,0)$.

Define the map $T: X \rightarrow C B^{\sigma}(X)$ by

$$
T 0=T 1=\{0\}, \quad T 2=\{0,1\}
$$

Note that it easy that $T x$ is bounded and is closed for all $x \in X$ in the dislocated metric space $(X, \sigma)$.

We shall show that

$$
H_{\sigma}(T x, T y) \leq \frac{8}{11} M(x, y), \quad \forall x, y \in X .
$$

For this, we distinguish the following cases:

case $1: x, y \in\{0,1\}$. We have

$$
H_{\sigma}(T x, T y)=\sigma(0,0)=0 \leq \frac{8}{11} \sigma(x, y) \leq \frac{8}{11} M(x, y) .
$$

case $2: x \in\{0,1\}, y=2$. We have

$$
\begin{aligned}
H_{\sigma}(T x, T y)=H_{\sigma}(\{0\},\{0,1\}) & =\max \{\sigma(0,\{0,1\}), \max \{\sigma(0,0), \sigma(0,1)\}\} \\
& =\max \left\{\min \{\sigma(0,0), \sigma(0,1)\}, \frac{1}{3}\right\}=\frac{1}{3}
\end{aligned}
$$




$$
\leq \frac{8}{11} \sigma(x, y) \leq \frac{8}{11} M(x, y)
$$

$\underline{\text { case } 3}: x=y=2$. We have

$$
\begin{gathered}
H_{\sigma}(T x, T y)=H_{\sigma}(\{0,1\},\{0,1\})=\max \{\sigma(0,\{0,1\}), \sigma(1,\{0,1\})\} \\
=\min \{\sigma(0,1), \sigma(1,1)\}=0 \leq \frac{8}{11} \sigma(2,2) \leq \frac{8}{11} M(2,2) .
\end{gathered}
$$

Thus, all the required hypotheses of Theorem 2 are satisfied. Then, $T$ has a fixed point. Here, $x=0$ is the unique fixed point of $T$.

Example 5. Let $X=\{0,1,2\}$ and $\sigma: X \times X \rightarrow[0, \infty)$ defined by

$$
\begin{aligned}
& \sigma(0,0)=0, \sigma(1,1)=3, \sigma(2,2)=1 \\
& \sigma(0,1)=\sigma(1,0)=7, \quad \sigma(0,2)=\sigma(2,0)=3 \quad \text { andquad } \sigma(1,2)=\sigma(2,1)=4 .
\end{aligned}
$$

Then, $(X, \sigma)$ is a complete dislocated metric space. Note that $\sigma$ is not a partial metric on $X$ because $\sigma(0,1) \geq \sigma(2,0)+\sigma(2,1)-\sigma(2,2)$.

Define the map $T: X \rightarrow C B^{\sigma}(X)$ by

$$
T 0=T 2=\{0\} \quad \text { and } T 1=\{0,2\} .
$$

Note that $T x$ is bounded and is closed for all $x \in X$ in the dislocated metric space $(X, \sigma)$.

We shall show that

$$
H_{\sigma}(T x, T y) \leq \frac{3}{4} M(x, y), \quad \forall x, y \in X .
$$

For this, we consider the following cases:

case 1: $\quad x, y \in\{0,2\}$. We have

$$
H_{\sigma}(T x, T y)=\sigma(0,0)=0 \leq \frac{3}{4} M(x, y) .
$$

case2: $x \in\{0,2\}, y=1$. We have

$$
\begin{aligned}
H_{\sigma}(T x, T y) & =H_{\sigma}(\{0\},\{0,2\})=\max \{\sigma(0,\{0,2\}), \max \{\sigma(0,0), \sigma(0,2)\}\} \\
& =\max \{0,3\}=3 \leq \frac{3}{4} \sigma(x, y) \leq \frac{3}{4} M(x, y) .
\end{aligned}
$$

case 3: $\quad x=y=1$. We have

$$
\begin{aligned}
H_{\sigma}(T x, T y) & =H_{\sigma}(\{0,2\},\{0,2\})=\max \{\sigma(0,\{0,2\}), \sigma(2,\{0,2\})\} \\
& =\min \{\sigma(0,2), \sigma(2,2)\}=1 \leq \frac{3}{4} \sigma(1,1) \leq \frac{3}{4} M(1,1) .
\end{aligned}
$$

Therefore, all the required hypotheses of Theorem 2 are satisfied. Here, $x=0$ is the unique fixed point of $T$ 
Example 6. Let $X=[0,1]$ and $\sigma: X \times X \rightarrow[0, \infty)$ defined by

$$
\sigma(x, y)=x+y, \quad \forall x, y \in X
$$

Then, $(X, \sigma)$ is a complete dislocated metric space. Note that $\sigma$ is not a partial metric on $X$ because $\sigma(x, x)>\sigma(x, y)$ for all $x>y$. $\sigma$ is not also a metric on $X$ since $\sigma(1,1)=2$.

Define the map $T: X \rightarrow C B^{\sigma}(X)$ by

$$
T x=\left\{0, \frac{x^{2}}{1+x}\right\}, \quad \forall x \in X
$$

It is easy that $T x$ is bounded and is closed for all $x \in X$ in the dislocated metric space $(X, \sigma)$.

We shall show that

$$
H_{\sigma}(T x, T y) \leq \frac{1}{2} M(x, y), \quad \forall x, y \in X .
$$

For this, we consider the following cases:

case1: $\quad x=y$. We have

$$
\begin{aligned}
H_{\sigma}(T x, T y) & =\max \left\{\sigma(0, T x), \sigma\left(\frac{x^{2}}{1+x}, T x\right)\right\} \\
& =\max \left\{\min \left\{\sigma(0,0), \sigma\left(0, \frac{x^{2}}{1+x}\right)\right\}, \min \left\{\sigma\left(0, \frac{x^{2}}{1+x}\right), \sigma\left(\frac{x^{2}}{1+x}, \frac{x^{2}}{1+x}\right)\right\}\right\} \\
& =\max \left\{0, \frac{x^{2}}{1+x}\right\}=\frac{x^{2}}{1+x} \leq x=\frac{1}{2} \sigma(x, x) \leq \frac{1}{2} M(x, y) .
\end{aligned}
$$

case2: $\quad x \neq y$. Since $\sigma$ is symmetric, we suppose $x>y$. We have

$$
\begin{aligned}
& H_{\sigma}(T x, T y) \\
&= H_{\sigma}\left(\left\{0, \frac{x^{2}}{1+x}\right\},\left\{0, \frac{y^{2}}{1+y}\right\}\right) \\
&= \sup \left\{\max \left\{\sigma\left(0,\left\{0, \frac{y^{2}}{1+y}\right\}\right), \sigma\left(\frac{x^{2}}{1+x},\left\{0, \frac{y^{2}}{1+y}\right\}\right)\right\},\right. \\
&\left.\max \left\{\sigma\left(0,\left\{0, \frac{x^{2}}{1+x}\right\}\right), \sigma\left(\frac{y^{2}}{1+y},\left\{0, \frac{x^{2}}{1+x}\right\}\right)\right\}\right\} \\
&\left.\left.=\max \left\{\sigma\left(\frac{x^{2}}{1+x},\left\{0, \frac{y^{2}}{1+y}\right\}\right)\right\}, \sigma\left(\frac{y^{2}}{1+y},\left\{0, \frac{x^{2}}{1+x}\right\}\right)\right\}\right\} \\
&=\max \left\{\frac{x^{2}}{1+x}, \frac{y^{2}}{1+y}\right\}=\frac{x^{2}}{1+x} \leq \frac{1}{2} x \leq \frac{1}{2}(x+y)=\frac{1}{2} \sigma(x, y) \leq \frac{1}{2} M(x, y) .
\end{aligned}
$$

Thus, all the required hypotheses of Theorem 2 are satisfied. Here, $x=0$ is the unique fixed point of $T$. 
Now, we provide an application on the research of a solution of an integral equation. For instance, using Corollary 6, we will prove the existence of a solution of the following integral equation.

$$
x(t)=\int_{a}^{b} K(t, x(s)) d s
$$

where $K:[a, b] \times \mathbb{R} \rightarrow[0, \infty)$ is a continuous nonnegative function.

Throughout this part, let $X=C([a, b],[0, \infty))$ be the set of real nonnegative continuous functions defined on $[a, b]$. Take the dislocated metric $\sigma: X \times X \rightarrow[0, \infty)$ defined by

$$
\sigma(x, y)=\|x\|_{\infty}+\|y\|_{\infty}=\max _{s \in[a, b]} x(s)+\max _{s \in[a, b]} y(s) \quad \text { for all } x, y \in X .
$$

Mention that $\sigma$ is not partial metric on $X$. But, it is easy that $(X, d)$ is a complete dislocated metric space.

Now, take the operator $T: X \rightarrow X$ defined by

$$
T x(t)=\int_{a}^{b} K(t, x(s)) d s .
$$

Mention that (4.1) has a solution if and only if the operator $T$ has a fixed point.

The main result is

Theorem 3. Assume that there exists $\lambda \in(0,1)$, such that for every $s \in[a, b]$ and $u \in X$, we have

Then, $T$ has a fixed point in $X$

$$
K(s, u(s)) \leq \frac{\lambda}{b-a} u(s) .
$$

Proof. For all $x \in X$

$$
\begin{aligned}
|T(x)(t)| & \leq \int_{a}^{b}|K(t, s, x(s))| d s \\
& \leq \frac{\lambda}{b-a} \int_{a}^{b} x(s) d s \leq \lambda\|x\|_{\infty} .
\end{aligned}
$$

It follows that for all $x, y \in X$

$$
\sigma(T x, T y) \leq \lambda \sigma(x, y) \leq \lambda M(x, y) .
$$

Therefore, all the hypotheses of Corollary 6 are satisfied. Consequently, $T$ has a fixed point, that is, (4.1) has a solution $x \in X$.

\section{ACKNOWLEDGEMENT}

The authors express their gratitude to the referees for constructive and useful remarks and suggestions. 


\section{REFERENCES}

[1] C. T. Aage and J. N. Salunke, "The results on fixed points in dislocated and dislocated quasi-metric space," Appl. Math, vol. 2, no. 59, pp. 2941-2948, 2008.

[2] S. Al-Mezel, C. M. Chen, E. K. nar, and V. Rakocević, "Fixed point results for various $\alpha$ admissible contractive mappings on metric-like spaces," Abstract and Applied Analysis, no. Article ID 379358, 2014.

[3] H. H. Alsulami, E. nar, and H. Piri, "Fixed points of modified $f$-contractive mappings in complete metric-like spaces," Journal of Function Spaces, no. Article ID 270971, 2015.

[4] H. Aydi, M. Abbas, and C. Vetro, "Partial hausdorff metric and nadler's fixed point theorem on partial metric spaces," Topology and its Appl., vol. 159, pp. 3234-3242, 2012.

[5] H. Aydi, M. Abbas, and C. Vetro, "Common fixed points for multivalued generalized contractions on partial metric spaces," RACSAM - Revista de la Real Academia de Ciencias Exactas, Fisicas y Naturales. Serie A. Matematicas, vol. 108, pp. 483-501, 2014.

[6] H. Aydi and E. Karapınar, "Fixed point results for generalized $\alpha-\psi$-contractions in metric-like spaces and applications," Electronic Journal of Differential Equations, vol. 2015, no. 133, pp. $1-15,2015$.

[7] R. D. Daheriya, R. Jain, and M. Ughade, "Some fixed point theorem for expansive type mapping in dislocated metric space," ISRN Math. Anal., no. Article ID 379358, 2012.

[8] R. George, "Cyclic contractions and fixed points in dislocated metric spaces," Int. J. Math. Anal., vol. 7, no. 9, pp. 403-411, 2013.

[9] A. A. Harandi, "Metric-like spaces, partial metric spaces and fixed points," Fixed Point Theory Appl., vol. 204, 2012.

[10] G. E. Hardy and T. D. Rogers, "A generalization of a fixed point theorem of reich," Canadian Mathematical Bulletin, vol. 16, pp. 201-206, 1973.

[11] P. Hitzler, "Generalized metrics and topology in logic programming semantics," Ph.D. dissertation, School of Mathematics, Applied Mathematics and Statistics, National University Ireland, University College Cork, 2001.

[12] P. Hitzler and A. K. Seda, "Dislocated topologies," J. Electr. Eng, vol. 51, no. 12, pp. 3-7, 2000.

[13] A. Isufati, "Fixed point theorems in dislocated quasi-metric space," appl," Math. Sci, vol. 4, no. 5, pp. $217-233,2010$.

[14] R. Kannan, "Some results on fixed points," Bulletin of the Calcutta Mathematical Society, vol. 10, pp. 71-76, 1968.

[15] P. S. Kumari, "Some fixed point theorems in generalized dislocated metric spaces," Math. Theory Model., vol. 1, no. 4, pp. 16-22, 2011.

[16] P. S. Kumari, W. Kumar, and I. R. Sarma, "Common fixed point theorems on weakly compatible maps on dislocated metric spaces," Math. Sciences, vol. 6, no. 71, pp. 1-5, 2012.

[17] S. G. Matthews, "Partial metric topology," in Proceedings of the 8th Summer Conference on General Topology and Applications, vol. 728, , Annals of the Academy of Sciences, 1994, pp. 183-197.

[18] S. B. Nadler, "Multivalued contraction mappings," Pacific J. Math., vol. 30, pp. 475-488, 1969.

[19] E. K. nar and P. Salimi, "Dislocated metric space to metric spaces with some fixed point theorems," Fixed Point Theory Appl., vol. 222, 2013.

[20] I. R. Sarma and P. S. Kumari, “On dislocated metric spaces,” Int. J. Math. Arch., vol. 3, no. 1, pp. 72-77, 2012.

[21] M. Shrivastava, K. Qureshi, and A. D. Singh, "A fixed point theorem for continuous mapping in dislocated quasi-metric spaces," Int. J. Theor. Appl. Sci., vol. 4, no. 2, pp. 39-40, 2012.

[22] R. Shrivastava, Z. K. Ansari, and M. Sharma, "Some results on fixed points in dislocated and dislocated quasi-metric spaces," J. Adv. Stud. Topol., vol. 3, no. 1, pp. 25-31, 2012. 
[23] K. Zoto, "Some new results in dislocated and dislocated quasi-metric spaces," Appl. Math. Sci., vol. 6, no. 71, pp. 3519-3526, 2012.

Authors' addresses

H. Aydi

University of Dammam Department of Mathematics. College of Education of Jubail, P.O: 12020 ., 31961 Industrial Jubail, Saudi Arabia, and Department of Medical Research, China Medical University Hospital, China Medical University, Taichung, Taiwan

E-mail address: hmaydi@uod.edu.sa

\section{A. Felhi}

KF University, College of Sciences. Department of Mathematics, Al-hasa, Saudi Arabia Current address: Université de Carthage, Institut Préparatoire de Bizerte, Jarzouna, Tunisie E-mail address: afelhi@kfu.edu.sa

\section{Erdal Karapınar}

Atilim University, Department of Mathematics 06836, İncek Ankara, Turkey

E-mail address: erdalkarapinar@yahoo.com

\section{S. Sahmim}

KF University, College of Sciences. Department of Mathematicst, Al-hasa, Saudi Arabia

Current address: Université de Carthage, Laboratoire D’Ingénirie Mathématiques, Ecole Polytechniques de Tunisie, P.B.743-2078, La Marsa, Tunisie

E-mail address: ssahmim@kfu.edu.sa 